\title{
Brownian Distance Correlation-Directed Search: a fast feature selection technique for Alternate Test
}

\author{
Gildas Leger $^{\mathrm{a}}$, Manuel J. Barragan ${ }^{\mathrm{b}, \mathrm{c}}$ \\ ${ }^{a}$ Instituto de Microlectrónica de Sevilla, CSIC-Universidad de Sevilla, Av. Américo Vespucio s/n, \\ 41092 Sevilla, Spain \\ ${ }^{b}$ CNRS, TIMA, F-38000 Grenoble, France \\ ${ }^{c}$ Université Grenoble Alpes, TIMA, F-38000 Grenoble, France
}

\begin{abstract}
Machine-learning indirect test relies on powerful statistical algorithms to build prediction models that relate cheap measurements to costly performance metrics. Though many works in the past have been focused on proposing different models or on ways to improve the reliability of the results, it appears that the main bottleneck of the approach is the definition of an information-rich input space. Finding the appropriate measurements that are both cheap and meaningful is a task that has not yet been automated. In this framework, feature selection is a necessary tool to explore possible candidates. In this paper a hybrid method is proposed that lay between filtering and wrapper-based methods, trying to strike the right balance between accuracy and speed for the particular case of Alternate Test.
\end{abstract}

Keywords: AMS-RF test, Alternate Test, Machine-learning, Feature selection

\section{Introduction}

For AMS-RF circuits, specification-based testing is the golden standard. This is not surprising since the data-sheet with the specifications is actually what is sold to the customer. In addition, specification-based testing has an edge over defect-oriented methods: it does not require any sort of prior validation. Defect-oriented methods, extensively used for digital circuits, rely on the assumption that the circuit is correct by design and look for deviations from the expected behavior. On the contrary, specificationbased test would catch any cause of malfunction, even a design error. Thus, we may wonder why specification-based has even been challenged in this application domain. The reason is cost. Measuring performance of state-of-the art products in a production environment is a daunting task. Moreover, exhaustive validation is prohibitive for most AMS-RF circuits of reasonable complexity. It requires high-end testers and long test times.

Email addresses: leger@imse-cnm.csic.es (Gildas Leger), manuel.barragan@imag.fr (Manuel J. Barragan) 
Machine-learning indirect test [1] is a bridge between defect-oriented solutions, which have a low-cost potential, and specification-based test. Being an alternative to conventional testing it is also known as Alternate Test. The basic idea is to perform simple (and cheap) measurements on the circuit under test, and then use modern machine learning algorithms to map these simple measurements onto the specification space. In order to build the mapping, both simple and performance measurements have to be acquired for a small population of circuits which is called the training set. This information is used to calibrate the parameters of the regression model; a process called supervised learning. For the rest of the population, only the simple measurements need to be performed and the performance is inferred using the learned models, driving test cost down. These simple measurements are often called signatures if they are considered from the perspective of a designer or a test engineer but they are called features when considered from the viewpoint of a statistician or a data engineer. Since the selection method that is described in this paper could easily be transposed to other fields than electronic testing, the term "feature" will be preferred in what follows.

One of the key aspects that, in our opinion, limits the adoption of Alternate test as a methodology is the definition of the input space of features. Information-rich features, that are sensitive to the performance degradation mechanisms, are required. While only the main degradation mechanisms may lead to sufficient prediction accuracy, reliability requirements may rise the standards and require a much more complete input space.

Moreover, if relevant information is missing from the input feature space, it may lead to non-model errors. As an example, we can refer a recent paper that shows that the wafer fabrication process using a stepper induces patterned variations across it. The authors define similarity clusters (also by machine-learning) and train specific models for each cluster [2]. This implicitly means that the localization of the circuit on the wafer matters in the Alternate Test approach, and should thus be taken as an input.

Unfortunately, we are not aware of any generic method to build a complete and information-rich set of features in a top-down approach. Provided a test target (the prediction of a given specification with a given accuracy) there is no mean, at present, to propose a set of simple measurements that will meet this target. The current approach for designing features is mostly ad-hoc and heavily relies on the expertise of the designer. It must be acknowledged that some works have been presented to optimize a particular kind of stimulus and extract appropriate data from the circuit response with the purpose of building a regression model. For instance in [3], a piecewise linear waveform is used as a test stimulus and the time-amplitude coordinates are optimized with Genetic Algorithms. A gradient search algorithm is also used in [4] for selecting the tones of a multi-tone RF stimulus. Still, both these papers are dedicated to specific circuits and cannot be considered a generic methodology since the type of stimulus is chosen ad-hoc.

Most of the time, a bottom-up approach has thus to be contemplated: a large number of feature candidates are proposed and a selection is performed to keep only the most relevant subset. In the field of statistics, this is known as feature selection. Let us consider an example. Alternate Test was originally proposed for RF circuits. Clean RF signal generation and acquisition is not an easy task and the perspective of substituting $\mathrm{RF}$ measurements by baseband or even DC features was seducing. The DC operating points and quiescent currents of the different nodes and branches of the circuits under test are thus usual feature candidates [5]. For circuits of medium complexity, this readily represents a significant search space. Repeating the measurements under different operating 
conditions (like the supply voltage or the operating frequency) to stress the circuit and extract additional information can further increase the dimensionality of the candidate space [6].

In such a context, were hundreds of feature candidates may be available, efficient feature selection tools are necessary to explore the input space. Efficient, here, means that the accuracy of model trained with the selected features (given possible selection constraints of the model, like the maximum number of features) must be as close as possible to the optimum accuracy obtained with the best possible subset. But efficient also means that the selection must be performed in a reasonable amount of time.

For this purpose, this work proposes a new method that can be called Brownian Distance Correlation-Directed Search. The paper is organized as follows: The first section conceptually describes some of the most representative approaches to feature selection and presents our proposal. Feature selection methods are usually classified in two families: The filtering approaches that are fast but with limited accuracy and the wrapper approaches that are accurate but slow. The proposed hybrid approach, as originally sketched in [7], pretends to take the best of both families and extend further the computational/accuracy trade-off. The second section presents some experimental validation results. For that purpose, five different cases of study are contemplated that cover different application segments. Finally the concluding section summarizes and discusses the benefits and drawbacks of the proposed approach.

\section{Feature selection}

Feature selection can be defined as the process of selecting a subset of relevant features for use in model construction. It is a recurrent problem in machine learning, and has been addressed by numerous researchers in the specialized literature. Interested readers can refer to [8] for an excellent introduction to the topic.

In summary, the way to find a solution to the problem of selecting relevant features depends on several parameters:

- The number of features $N_{\text {feat }}$ in the search space which determines its size $\left(2^{N_{\text {feat }}}\right)$

- The particular regression model (It appears obvious that a linear model may not select the same features as a neural network).

- The number of training samples which limits the number of selected features due to the Curse of Dimensionality (As sample density decreases with dimensionality, it is well known that regression models fail to properly discriminate between real relevant features and noise).

- The information relevance of the features. The information carried out by a given feature can be relevant or not depending on the presence or absence of other features in the input space.

One important thing to notice is that Statistics literature almost always addresses feature selection from the viewpoint of overfitting and model performance. That is to say: dimensionality reduction is usually the main concern. Indeed, training a model with 
too many features is detrimental since it ultimately tends to identify noise as relevant patterns.

However, in the particular application case of IC testing there is a specific additional concern: each extra feature corresponds to an additional measurement that must be performed in production which increases test time. In the field of test, feature selection has actually been used before the apparition of advanced machine-learning algorithms in the field of test compaction $[9,10]$. It was early acknowledged that the different specifications of a circuit are likely to be correlated and that it may not be worth measuring all of them. In a generic way, it is thus beneficial to reduce the number of features.

\subsection{Wrapper approach}

The wrapper approach consists in using the machine-learning prediction model as a black box within an optimization loop. The model is used to evaluate the prediction error (also called generalization error) for a given feature subset, and the optimization loop tries to minimize this error. An example of wrapper approach applied to test can be found in [11] in the context of classification.

Combinatorial optimization is a research field in itself, and there exist a plethora of approaches, from gradient descent to genetic algorithms. It is out of the scope of this paper to investigate which is the best suited for feature selection. By the way, it is more than probable that there is not a unique generic answer.

Wrapper approaches should give the most accurate results since the criterion to select or drop a feature is the model error: the same model that will be used to perform the regression in the Alternate Test procedure itself. There are significant pitfalls, though, to the use of wrapper approaches. One of them is that the optimization process itself is a machine-learning process. In this sense, it somehow competes with the embedded and implicit optimization loop of model training. The generalization error is evaluated on a test set that is different from the training set. However, if this generalization error is used as the cost function for the feature selection optimizer, the optimizer may end up selecting the features that are most suited for that particular test set. At some point, due to the finite sample size of the set, it is possible to fit the data with random noise variables. We thus need an out-of-the-loop criterion to ensure/verify that the model is not overfitting. We can implement a sanity check consisting in monitoring the model performance on an out-of-the loop validation set, to verify that the generalization error for this set also improves. The feature optimizer must be stopped if a significant divergence is found between the improvement of the generalization error computed on the test set and the generalization error computed on the validation set.

Another issue is that many modern machine-learning algorithms contemplate some form of embedded dimensionality reduction techniques. These techniques are not equivalent to feature selection: transformations of the input space through kernels, linear combinations of features, regularization penalties etc. They may not drop any feature but combine them to form more predictive ones. Though they are different, these techniques clearly compete with the feature selection optimizer and hence the impact of adding of a useless feature may not appear as negatively as it should on the generalization error.

But the most important issue is computational: training a state-of-the-art machinelearning model takes time. This time is a complex function of the model type, the number of features (the dimensionality of the input space) and the number of training 
Table 1: Training time for different model ensembles

\begin{tabular}{lccc}
\hline \# of features & 5 & 10 & 20 \\
\hline \# of samples & 300 & & \\
\hline Perceptron & $7 \mathrm{~s}$ & $48 \mathrm{~s}$ & $3 \mathrm{mn}$ \\
Nearest Neighbors & $8 \mathrm{~s}$ & $18 \mathrm{~s}$ & $28 \mathrm{~s}$ \\
MARS & $1 \mathrm{mn} 40 \mathrm{~s}$ & $4 \mathrm{mn}$ & $9 \mathrm{mn} 40 \mathrm{~s}$ \\
Radial Basis Function & $12 \mathrm{~s}$ & $13 \mathrm{~s}$ & $17 \mathrm{~s}$ \\
\hline \# of samples & 1000 & & \\
\hline Perceptron & $15 \mathrm{~s}$ & $1 \mathrm{mn} 6 \mathrm{~s}$ & $3 \mathrm{mn} 15 \mathrm{~s}$ \\
Nearest Neighbors & $34 \mathrm{~s}$ & $1 \mathrm{mn} 40 \mathrm{~s}$ & $3 \mathrm{mn} 7 \mathrm{~s}$ \\
MARS & $7 \mathrm{mn} 30 \mathrm{~s}$ & $19 \mathrm{mn} 40 \mathrm{~s}$ & $47 \mathrm{mn} 13 \mathrm{~s}$ \\
Radial Basis Function & $1 \mathrm{mn} 27 \mathrm{~s}$ & $1 \mathrm{mn} 50 \mathrm{~s}$ & $2 \mathrm{mn}$ \\
\hline \# of samples & 10000 & & \\
\hline Perceptron & $4 \mathrm{mn} 25 \mathrm{~s}$ & $8 \mathrm{mn} 20 \mathrm{~s}$ & $16 \mathrm{mn} 50 \mathrm{~s}$ \\
Nearest Neighbors & $19 \mathrm{mn} 15 \mathrm{~s}$ & $1 \mathrm{~h} 32 \mathrm{mn}$ & $2 \mathrm{~h} 28 \mathrm{mn}$ \\
MARS & $1 \mathrm{~h}$ & $2 \mathrm{~h} 10 \mathrm{mn}$ & $7 \mathrm{~h} 20 \mathrm{mn}$ \\
Radial Basis Function & $4 \mathrm{~h} 40 \mathrm{mn}$ & $12 \mathrm{~h} 55 \mathrm{mn}$ & $15 \mathrm{~h} 40 \mathrm{mn}$ \\
\hline
\end{tabular}

samples. To illustrate this fact, Table 1 shows the training time for four different models taken from the ENTOOL toolbox [12] (we assume that all models are encoded with the same efficiency): Perceptron Neural Network, Nearest Neighbors, Multivariate Adaptive Regression Splines and Radial Basis Function networks. These models were trained over 300, 1000 and 10000 samples for 5, 10 and 20 input features. In each case, the final models are actually ensemble models that average the results of the 9 best models out of 15 (trained on 15 data partitions with non-overlapping test sets in a cross-validation fashion). A state-of-the-art procedure would actually build an heterogeneous ensemble, considering different models on each data partition. For such an ensemble, the total training time would be the sum of the training times of the constituting models shown in Table 1.

It can be seen that the training computational burden of different models scales differently with respect to the dimensionality and the number of samples. In what follows, we will consider the use of a perceptron neural network which is the model type with lower computational burden in average (while maintaining a good accuracy).

A particularly simple algorithm for wrapper approach consists in a stepwise search, sketched in Fig. 1. This approach is based on a combination of stepwise forward addition and backward elimination. Starting from an initial subset of features, it explores all the possible children obtained by removing one existing feature or adding one new feature. At each iteration of the search, a number of new models are trained that depends on the already visited states of the search tree, but is approximately equal to the number of features in the search space, $N_{\text {feat }}$.

The choice of the starting point will give different outcomes. If we consider the complete set of available features as the initial set of features, the search will be geared toward 


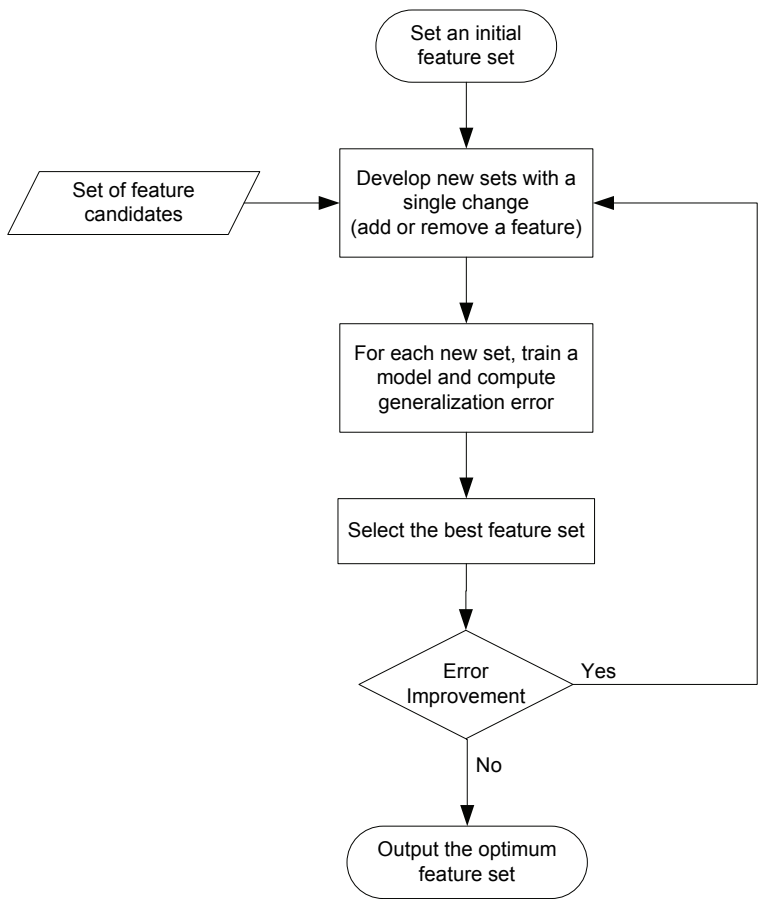

Figure 1: Flow diagram for stepwise search wrapper approach to feature selection

backward elimination. This is the case in [13] where the focus is put on test compaction, starting from a known good set of tests that the authors want to reduce. However, in a more generic case where a large space is to be explored, such a backward elimination may lead to an outcome with more features than necessary, due to the embedded regularization mechanisms of the machine-learning model itself, as discussed above. Taking a void set as the initial search point gears the search toward forward addition, which should lead to more compact sets. The drawback is that it may be more difficult to select groups of features that are significant together but not individually. Another possibility would consist in performing this search from different random seeds with an arbitrary (but reasonable) number of features. Obviously, the higher the number of seeds, the higher the computational cost.

To speed-up a bit the convergence, Kohavi has proposed in [14] to consider, at each iteration, the compound operators formed by the best children. In this case, it may be possible to add (or remove) several features in a single step if this provides a better model. However, our intention is to get a result close to the optimum front, considering an incremental number of features. For that purpose, it is better not to consider these compound operators and start the search from the void set. To get an idea of the variability of the possible models, we also perform a random search constraining the total number of features. In this way, we can get a cloud of points whose minimum should be close to the optimum front.

Obviously, genetic algorithms are known to guarantee convergence to the optimum solution. However, the speed of this convergence is unpredictable and it may end up 
requiring an even longer time (depending on the initial population size and the number of generations needed before convergence). Needless to say, only if the number of features is small would a full-search be feasible. For our comparison purpose, stepwise search will thus be considered as the reference in terms of accuracy and the produced fronts as the optimum fronts.

\subsection{The filtering approach}

On the other end of the feature selection spectrum, filtering approaches select adequate features without training any regression model. This is particularly relevant in situations where the number of feature candidates is extremely high. In such cases, they can be viewed as a pre-processing step to wrapper search discarding the less promising features to reduce the cardinality of the search space to a manageable size.

A wide variety of filtering approaches can be found in specialized statistics literature dedicated to dimensionality reduction [15] or feature selection [16]. It is impossible to cover all the methodologies that have been proposed. Instead, in this work we will compare different filtering strategies based on correlation and a recently published method called minimal-redundancy maximal-relevance criterion [17]. In addition, we will also comment the use of Principal Component Analysis (PCA) which is not strictly speaking a feature selection method but rather a dimensionality reduction technique. Despite of this, the reason why we include it into this study is because it is a widely-used preprocessing step in machine-learning and has actually been applied to Alternate Test [18].

\subsubsection{Correlation with performance}

A straightforward approach to supervised filtering would be to select the features that best correlate to the target specification. However, redundancy is an issue: if two features are almost identical, they could both be selected while only one bring original information.

In [19], it was proposed to use Brownian Distance correlation (dCor) instead of Pearson's correlation. The distance correlation statistics is defined in [20]. It is generated as follows: For a random sample $(\mathbf{X}, \mathbf{Y})=\left\{\left(X_{k}, Y_{k}\right): k=1, \ldots, n\right\}$ of $n$ i.i.d. random vectors $(X, Y)$ from the joint distribution of random vectors $X$ in $\mathbb{R}^{p}$ and $Y$ in $\mathbb{R}^{q}$, compute the Euclidian distance matrices $\left(a_{k l}\right)=\left(\left|X_{k}-X_{l}\right|_{p}\right)$ and $\left(b_{k l}\right)=\left(\left|Y_{k}-Y_{l}\right|_{q}\right)$. Define

$$
A_{k l}=a_{k l}-\bar{a}_{k .}-\bar{a}_{. l}+\bar{a}_{. .} \quad k, l=1, \ldots, n
$$

where

$$
\bar{a}_{k .}=\frac{1}{n} \sum_{l=1}^{n} a_{k l}, \quad \bar{a}_{\cdot l}=\frac{1}{n} \sum_{k=1}^{n} a_{k l}, \quad \bar{a}_{. .}=\frac{1}{n^{2}} \sum_{k, l=1}^{n} a_{k l}
$$

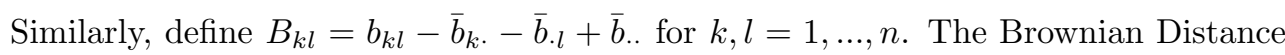
Covariance $\mathcal{V}_{n}(\mathbf{X}, \mathbf{Y})$ and Brownian Distance Correlation $\mathcal{R}_{n}(\mathbf{X}, \mathbf{Y})$ are then given by

$$
\mathcal{V}_{n}^{2}(\mathbf{X}, \mathbf{Y})=\frac{1}{n^{2}} \sum_{k, l=1}^{n} A_{k l} B_{k l}
$$


and

$$
\mathcal{R}_{n}^{2}(\mathbf{X}, \mathbf{Y})= \begin{cases}\frac{\mathcal{V}_{n}^{2}(\mathbf{X}, \mathbf{Y})}{\sqrt{\mathcal{V}_{n}^{2}(\mathbf{X}) \mathcal{V}_{n}^{2}(\mathbf{Y})}} & \mathcal{V}_{n}^{2}(\mathbf{X}) \mathcal{V}_{n}^{2}(\mathbf{Y})>0 \\ 0 & \mathcal{V}_{n}^{2}(\mathbf{X}) \mathcal{V}_{n}^{2}(\mathbf{Y})=0\end{cases}
$$

respectively, where the variance is given by

$$
\mathcal{V}_{n}^{2}(\mathbf{X})=\mathcal{V}_{n}^{2}(\mathbf{X}, \mathbf{X})=\frac{1}{n^{2}} \sum_{k, l=1}^{n} A_{k l}^{2}
$$

Distance correlation satisfies $0 \leq \mathcal{R} \leq 1$, and $\mathcal{R}=0$ only if $X$ and $Y$ are independent. In the bivariate normal case, $\mathcal{R}$ is a deterministic function of $\rho$, and $\mathcal{R}(X, Y) \leq|\rho(X, Y)|$ with equality when $\rho= \pm 1$.

Notice that the computation of Brownian Distance Correlation is quadratic in the number of samples. This may lead to large computation time on rich training sets. However, if we are looking for global trends -which is implicitly the case since Brownian Distance correlation is not local but acts on the complete variation range- it is possible to restrict the computation to a manageable subset of data points.

Compared to classical Pearson's correlation, the main advantage of Brownian Distance Correlation is its sensitivity to non-linear dependencies. Consider for instance two parameters that are deterministically related: $X$ is a uniform random variable in $[-1 ; 1]$ and $Y$ is expressed as $Y=e^{-10 X^{2}}$. For 1000 random samples, Pearson's correlation is 0.02 and Brownian Distance Correlation is 0.5. The former leads to think that the variables are uncorrelated while the latter clearly points at a dependency.

Another advantage of Brownian Distance Correlation is that it is multidimensional. Shortly put, the Brownian Distance Correlation computes the correlation between pairwise distances of samples in the two matrices under study. In this sense the dimensionality of the two matrices needs not be the same. For our case, we can thus correlate the pairwise distances between samples in the space of the selected features with the pair-wise distances in the vector of a given performance parameter.

Similarly to Brownian Distance Correlation, Kendall's Tau or rank correlation is usually used to assess variable independence and has also been used in the context of Alternate Test [21, 22]. It actually test the hypothesis that for correlated variables, pairwise sample variations should always evolve in the same direction. Hence,

$$
\tau=\frac{\sum_{i=1}^{n} \sum_{j=1}^{i}\left[\left(x_{i}<x_{j} \Rightarrow y_{i}<y_{j}\right) \text { OR }\left(x_{i}>x_{j} \Rightarrow y_{i}>y_{j}\right)\right]}{n(n-1) / 2}
$$

where $x$ and $y$ are the variables and $n$ is the number of samples. The logical expression in the numerator takes the value 1 if true and -1 if false. It can be seen that the philosophy is similar to Brownian Distance Correlation. However, the strength of the pairwise variations is not taken into account in Kendall's Tau which suggest a lower sensitivity. In addition, comparison between multidimensional spaces is impossible.

For Pearson's correlation and Kendall's Tau, the only solution is to rank the features as a function of their correlation to the performance. However, for Brownian Distance correlation we can also consider an iterative search increasing the dimensionality of the input space. We first select the feature that has the best univariate Brownian Distance 
correlation with the performance. This feature is thus included in the input space. At the second iteration we compute the multivariate Brownian Distance correlation between the performance and the two-dimensional input spaces formed by the first feature and the remaining candidate features.

\subsubsection{Minimal-redundancy maximal-relevance criterion}

Minimal-redundancy-maximal-relevance criterion (mRMR) is a recent and recognized filtering technique. It is a proxy of the maximal statistical dependency criterion based on mutual information [17]. As its name indicates, this technique aims at removing redundant features while keeping those that provide significant mutual information with the performance under study. It is a pure filtering approach, in the sense that it can be used to rank the features but does not provide per se an optimum size criterion.

In the documentation of their Matlab toolbox (the link for download is available in [17]), the authors indicate that the implementation of their algorithm works better on categorical data. For continuous datasets, they thus propose to discretize both the features and the performance under study. To do so, all variables are centered and divided by their respective standard deviations. Taking the closest integer of the results provides us with a categorical dataset.

\subsubsection{Principal Component Analysis}

The filtering techniques described above are supervised ones, in the sense that they make use of the target parameter (the performance) to select the features. However, one of the most used filtering approaches is actually an unsupervised one, that is, it acts only on the feature space: the so-called Principal Component Analysis (PCA).

When considering the full candidate feature space, in most cases all the features are not independent. If we could plot the samples on this multidimensional space, it may result that the cloud of point lays on a manifold of lower dimension. The goal of PCA is to determine this reduced manifold, supposing that it is linear in the feature space. In other words, it identifies the directions in the full candidate feature space that best explain the variation observed in the data. To do so, it performs an eigenvalue decomposition of the features covariance matrix. The Principal Components (PCs) are the eigenvectors that correspond to the largest eigenvalues.

While it may effectively circumvent the curse of dimensionality and solve the computational issue of model training, it does not necessarily reduce the number of features to be acquired. The eigenvectors that form the principal components are linear combinations of the original features. It may happen (and it usually does) that the first few principal components require the complete set of features for their computations.

Besides this practical test cost aspect, one of the of the main conceptual drawbacks of PCA is the fact that it is a linear method, while the relation between features may be non-linear.

\subsection{Proposed approach: Brownian Distance Correlation-Directed Search}

Filtering approaches are extremely fast but with low accuracy while wrapper approaches are slow but accurate. In order to get the best of both worlds, we propose the following directed search:

The idea behind this algorithm is the same as projection pursuit: a given feature may explain the bold behavior of the specification but tiny details look like noise with respect 


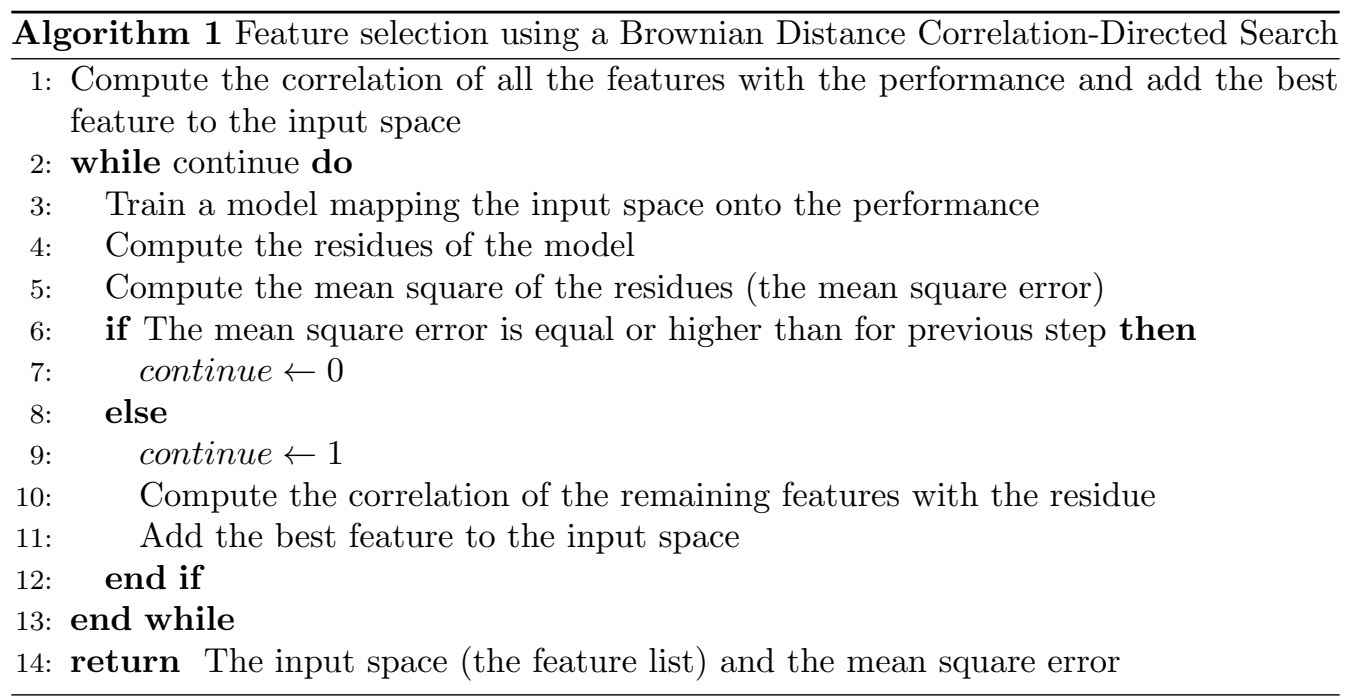

to the main contribution. However, instead of subtracting the projection of the selected feature from the performance measurement, which would work fine only for a linear problem, we rather train a model and compute the residues (i.e. subtracting the model prediction from the real values). The residues thus contain the non-modeled information. The next step of the algorithm (step 10 in algorithm 1) computes the distance correlation of the remaining feature candidates with the residues and selects as the most correlated as the next feature to be included in the search space.

This procedure acknowledges the essentially non-linear and multivariate relation between the feature space and the performance measurement. In that way, the next selected feature should always bring additional information. Redundant features should never be selected because their effects are already modeled and cannot be present in the residues.

A key point of the algorithm above is the concept of correlation. In this work we propose to use multivariate Brownian Distance correlation, as described in the Secion 2.2.1, instead of the conventional Pearson's correlation. If we were to use Pearson's correlation, we would be implicitly considering a linear problem. Assuming linear conditions is a huge restriction that may lead to incorrect feature selection decisions. The introduction of Brownian Distance Correlation, on the other hand, allows us to handle multivariate non-linear dependencies in order to direct the search of relevant features. The correlation is actually computed between the augmented input space - that is to say the matrix of previously selected features plus the feature candidate - and the vector of residues.

\subsection{Benefits and drawbacks}

\subsubsection{Computation time}

Let us imagine a worst case scenario where the maximum model accuracy is required. An heterogeneous ensemble with four model types is considered. Selecting the best 10 features out of 100 candidates using stepwise wrapper search would thus requires approximately 99 ensemble model trainings for each new feature from 1 to 10 . If we consider the training time for 5 features (i.e. the first column in Table 1) as the average 
training time - which is conservative since most models do not scale linearly with the number of features - the total time for stepwise wrapper search completion would be 1 day and 13 hours for 300 samples, 6 days and 14 hours for 1000 samples and 2 months and 17 days for 10000 samples. For a MARS model (which is the most widely used model for Alternate Test) the total time for stepwise wrapper search completion would be 1 day and 3 hours for 300 samples, 5 days for 1000 samples and 1 month and 11 days for 10000 samples.

In [23], a Genetic Algorithm is used to perform feature selection and explore the cost-performance trade-off of different subsets. However, the authors do not comment the time required to compute the optimum fronts in the worst case (50 generations with 286 chromosomes).

Depending on the situation, wrapper search may be feasible or not. In the ideal situation where all the features (which correspond to different measurements) are always available for the circuit under test, then the feature selection could be performed after closing the design. Whenever the exact accuracy of the Alternate Test solution does not need to be known before tape-out, wrapper approaches may be adequate. On the other hand, if feature selection has to be performed before tape-out (for instance because some feature require extra circuitry or more generally Design-for-Testability modifications of the chip ) then the designer would clearly prefer a fast solution to speed up decisionmaking. Indeed, if the target accuracy is not reached in a first attempt, more features could be proposed and several feature selection iterations may be necessary. A faster yet accurate feature selection method would thus be of great interest.

If we neglect the computation time of the correlation (in the case of the proposed approach) or mutual information (in the case of mRMR), an approximation of the computation cost is the number of models to be trained. For the mMRM filtering approach (and the rest of filtering methods), the ranking of features is almost instantaneous. However there is no criterion to decide the number of features to be used. As far as we know, the only solution consists in learning incremental models -iteratively adding new features in the ranking order-and supervising the evolution of the generalization error on an independent set. When the model accuracy does not improve, the iterative addition is stopped. The obtained computing burden is thus the same as for the proposed directed search approach, which also train models iteratively to compute the residues. In both cases, the number of models to be trained would be approximately $N_{\text {opt }}$.

The reduction factor in computational cost with respect to stepwise wrapper search is thus at least equal to $N_{\text {feat }}$. For cases more complex than those studied here, it could represent more than 2 orders of magnitude.

\subsubsection{Cost optimization}

Ideally feature selection should be seen as a cost optimization process. Model accuracy, which depends on the selected feature set, translates into test escapes and yield losses. Similarly, each feature corresponds to a measurement that has to be carried out in the production line and thus implies additional test time and/or resources. The overall test cost associated to a particular feature set is not, however, as simple as the sum of the costs associated to the individual measurements. Indeed, there are shared costs related to test setups. For instance, six DC measurements at the nominal supply voltage may be cheaper than two DC measurements performed at two different supply voltages. 
A meaningful optimization requires a well-defined cost function, but the definition of the cost function is extremely application-specific and even company-specific since it also depends on market considerations (profit margin, reliability target, etc). Indeed, it is difficult to accurately relate the cost of test escapes and yield loss and the cost of performing the test in a single cost function. From a generic perspective, it is thus better to consider the optimum front between test cost and test accuracy, as in [23].

Obviously, wrapper search could implement any other cost function than the simple generalization error. This is not the case for the proposed approach or for filtering approaches. However, if some features are clearly more costly than others, or if they can be acquired in a single test setup, one can imagine to group them into "cost classes" and apply the fast search several times considering an incremental cost class. Another possible application of the proposed approach would be to provide a fast starting point to the stepwise wrapper search in order to speed-up convergence to a satisfying cost-effective solution.

In the experimental validation section the objective is to thus to select a reduced number of features among a large number of candidates to provide a good model accuracy. The number of features can be seen as a proxy for the test cost and the generalization error of the regression model as a proxy for the cost associated to escapes and yield losses. In this sense, we will simply assess the performance of the feature selection mechanisms by looking at the evolution of the generalization error versus the number of features.

\section{Experimental validation}

In order to validate the proposed feature selection technique, we apply it to a number of cases of study covering a broad application range: RF, switched-capacitor, continuous time analog and a more system-level case. Additionally, we consider also a third-party data set to show the performance of the approach in a very different application context.

In all cases, we use a perceptron neural-network from the ENTOOL MATLAB toolbox for ensemble learning [12] as the machine-learning model to predict a given performance parameter. The available data is split between a training set and a validation set. As their name indicate, the training set is used to train the machine-learning model while the validation set is used to check the predicting power of the model on unknown samples.

The available data in the training set is split in 15 random fractions and 15 different models realizations are obtained by leaving one of the 15 fractions out of the actual training data. This left-out fraction is used as a test set to estimate the generalization error, in a classical cross-validation fashion. At the end of this process, an ensemble model is created: the 9 best performing models are selected and their outcome is averaged to get the ensemble model output. One advantage of this scheme is that the training set data is randomly shuffled at each training iteration, which reduces (but does not totally remove) the risk of overfitting during feature selection.

Notice that the validation set that is kept apart is not involved by any means in model training or feature selection algorithms. It is exclusively used to monitor the evolution of the generalization error in an independent manner. Indeed, it is a common procedural error to apply the feature selection criterion on the validation set. This is not a problem for filtering approaches since the list of candidates is sorted a-priori. But for wrapper approaches, the chosen optimizer always ends up optimizing the generalization error for 


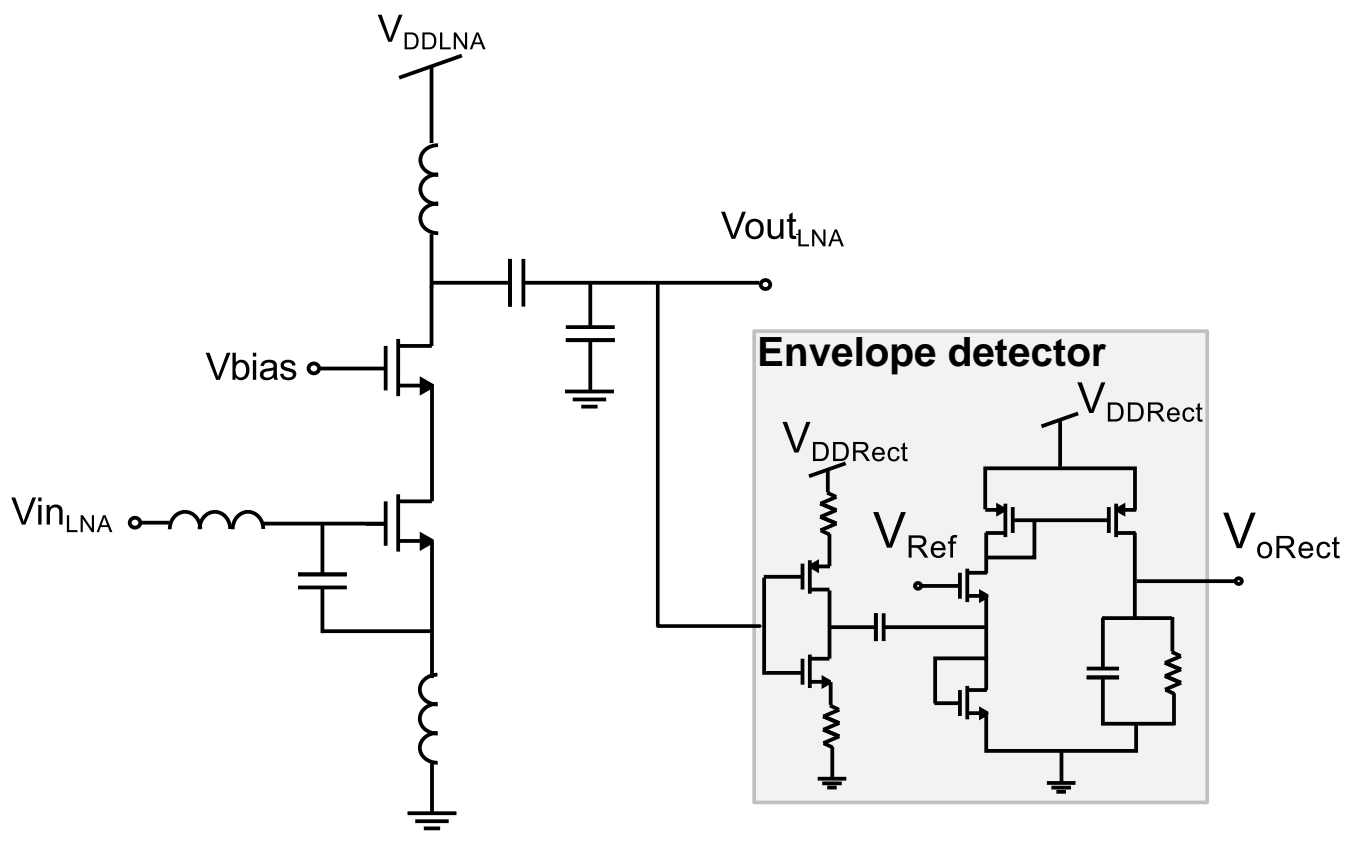

Figure 2: Schematic view of the LNA with envelope detector

that particular validation set, leading to overfitting. Unless otherwise stated, all the results in what follows are referred to this validation set and any improvement in the generalization error can be guaranteed to be due to feature information.

Notice that the different cases of study are circuits that were not necessarily thought to be tested by machine-learning indirect test. For some of them, only limited data is available either due to the cost of electrical performance simulation or simply because extensive Monte Carlo simulation was not considered necessary at design time. We will thus perform exhaustive comparisons only on the first case of study: an RF Low-Noise Amplifier for which significant data is available. From these we will identify the best filtering method and for the rest of cases of study, we will only compare the proposed method to the best filtering approach and to wrapper approach.

\subsection{RF Low Noise Amplifier}

\subsubsection{Circuit description}

The first case study is thus an RF Low Noise Amplifier. LNAs are simple circuits with very few components but that are quite sensitive to process variability. Both these aspects are valuable for our purpose since we need to perform many Monte Carlo simulations in order to build the training and testing sets of features and specifications. The variability is important to make the prediction more challenging.

Figure 2 shows the schematic of the LNA, which has been designed in a 90nm CMOS technology. The envelope detector at the output of the LNA has been included as a builtin test instrument [24]. This detector was intended to analytically retrieve specification information but can also be used in an Alternate Test framework. In particular we 
showed that it was necessary to include a mode that bypasses the LNA in such a way that the deviations due to the envelope detector alone can be accounted for.

Our initial set of candidates in this case study contains 42 features, consisting in the DC voltages in all the nodes of the LNA, the biasing current, the output of the envelope detector (with and without bypassing the LNA). All the previous features were measured under nominal power supply and also with supply stress (below nominal supply) since it was shown that this change in the operating conditions could bring additional information on the circuit. A population of 2000 instances of the LNA was generated using Monte Carlo simulation of the technology process parameters as stated in the design kit and 500 of them were set aside to form the validation set. For each instance, the 42 features as well as the gain of the LNA were measured.

The purpose is thus to select a minimum number of features that are able to predict the gain of the LNA. We acknowledge that the feasibility of the practical measurement is not addressed here. Adding a multiplexer to measure DC operating point may add unwanted parasitic loading to some critical node. Hence, once the feature selection is actually done an additional step would be required to correctly implement the required DfT modifications, as it was actually done for the envelope detector. Adding such a multiplexer may change the prediction accuracy of the model, but our goal is not to validate the Alternate Test itself. Notice that Notice that this fact by no means invalidates the feature selection procedure since all the methods make use of the same data.

\subsubsection{Comparing filtering methods}

In order to validate our approach, we will first show the limits of filtering approaches. For the sake of conciseness, we will consider only the first case study of the RF LNA and rank the 42 features according to:

- Pearson's Correlation to the LNA gain

- Kendall's Tau

- Univariate Brownian Distance Correlation to the LNA gain

- Multivariate Brownian Distance Correlation to the LNA gain

- Principal Component Analysis. Notice that in this case, all the features are actually used to compute the principal components.

- mRMR criterion

Figure 3 displays the obtained results -the different fronts up to a dimension of 10 together with the scatterplot obtained by wrapper search, to give an idea of the search space. Notice that the error values are computed on the verification set that has not been used elsewhere.

After identifying the best front from the wrapper search, we have repeated model training 30 times considering random data splits between training and validation sets. We thus obtain, for each point of the front, 30 different generalization errors from which we can extract an estimate of the confidence interval. The interval between the minimum and maximum error of the optimum front is depicted as a gray zone on the figure. 


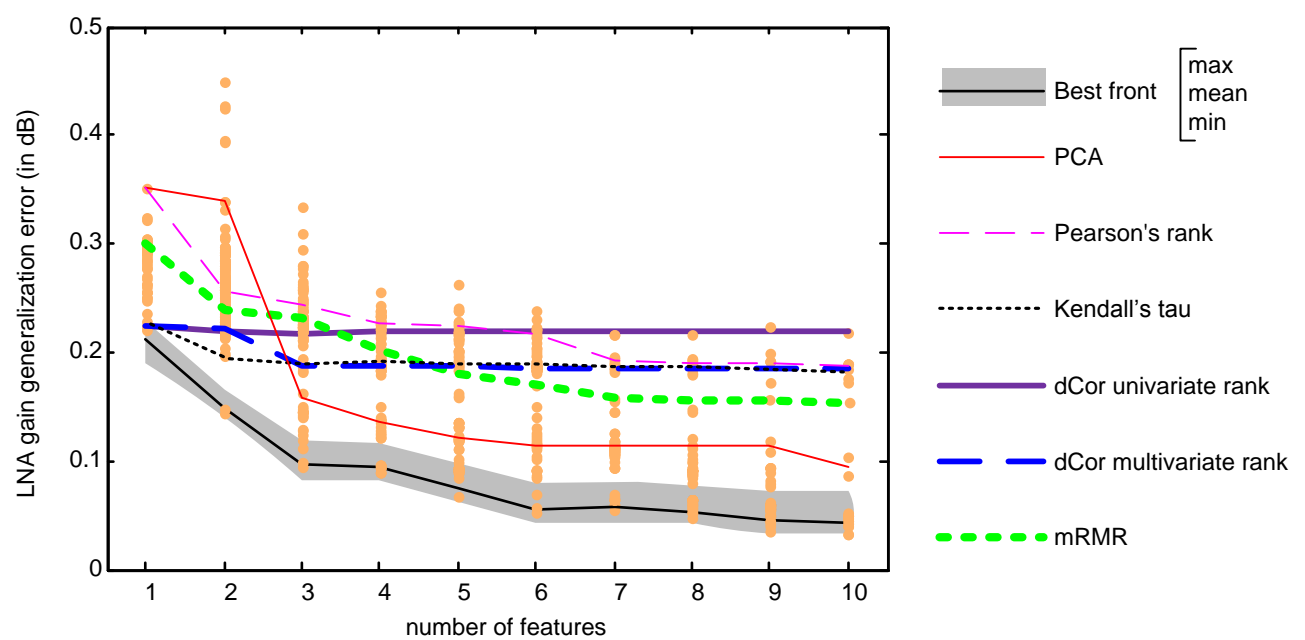

Figure 3: Generalization error for the prediction of LNA gain: Fronts obtained from different feature filtering methods and scatterplot of the explored space through wrapper search.

Table 2: Amount of variability concentrated in the first Principal Components

\begin{tabular}{lccccccc}
\hline \# of PCs $(n)$ & 1 & 2 & 3 & 4 & 5 & 6 & 7 \\
\hline Variability (in \%) & 75.6 & 96.9 & 99.0 & 99.82 & 99.93 & 99.98 & 99.99 \\
\hline
\end{tabular}

For correlation-based feature ranking, it appears that Kendall's Tau and Brownian Distance Correlation (both univariate and multivariate) accurately select the best first feature but fail to correctly identify the best additional features. Our guess (and this is what motivates our proposal) is that the redundant information after the selection of the first feature is not suppressed. Unsurprisingly, Pearson's correlation even fails to identify the best first feature, possibly due to non-linearity.

The behavior of the features ranked through mRMR criterion is correct, in the sense that the associated front shows a real improvement when adding new features. We can say that this method selects decently performing feature sets, but yet far from the optimum front.

The case of PCA is worth some dedicated comments. As it was said in Section 2.2.3, $\mathrm{PCA}$ is not a feature selection method but rather a dimensionality reduction technique. In this case, the X-axis legend in Fig. 3 must be understood as the dimensionality of the input space. That is, in the case of PCA it is the number of principal components (all of which are actually linear combinations of all the existing features). These principal components are designed to concentrate most of the variability information. Table 2 shows the percentage of the total input space variability explained by the first $n$ principal components. This is computed as the sum of the first $n$ eigenvalues of the input space covariance matrix over the total sum of eigenvalues. It can be seen that, in theory, the first principal component (i.e. the first eigenvector) concentrates more than $75 \%$ of the variability and the first two more than $96 \%$. It is thus expected that a model 
trained with the first few principal components should outperform any model trained with few genuine features because these few principal components contain the major part of the variability information. However, it can be seen in Fig. 3 that models trained with few (up to 10) principal components are worse than the models trained with the best set of genuine features. Models trained with the first or the first two Principal components are even worse than the those produced by the studied filtering methods. For a dimensionality above 3, PCA-based models never reach the optimum front (at least up to 10 principal components). We already knew that PCA was not a feature selection method but this experiment clearly shows that it is also far from optimum from the viewpoint of a dimensionality reduction technique. Using PCA for Alternate Test is thus a non-sense: not only would the PCA require to perform all the measurements but it does not guarantee to reach a precision close to the optimum. This is probably due to the fact that PCA implicitly considers that the correlations between features can be explained by linear relationships, which is clearly not the case here.

It can be concluded from this experiment that mRMR is the best of the studied filtering methods, in what follows we will consider only that particular filtering method to compare with our directed search approach and with the wrapper search for our different cases of study.

\subsubsection{Directed search with different correlation metrics}

As stated above, the underlying principle of the proposed directed search approach consists in subtracting the influence of the already selected features from the performance and select the features that best correlate with the generated residues. Here we compare the proposed algorithm that uses multivariate Brownian distance correlation to three variants that use Pearson's correlation, Kendall's tau and univariate Brownian distance correlation instead.

Figure 4 shows the obtained fronts for the RF LNA case study. As expected, the version that makes use of Pearson's correlation performs much worse than the proposed technique. More surprisingly, the version of the directed search with Kendall's Tau performs poorly. It manages to identify the first best feature but fails to find the best correlation with residues in further iterations. This is probably because Kendall's tau is more an independence test than a tool to measure the strength of the interactions between two variables.

The version with univariate Brownian distance correlation works well initially but appears to saturate for models that use more than 3 features. The improvement step when adding the ninth feature is meaningless and is probably casual: adding features can also lead to improvements. The fact that we see a plateau before this ninth feature instead of a degradation of the generalization error due to overfitting is probably due to the fact that the training algorithm of the perceptron model already implements some regularization technique and tends to null the coefficients of useless features.

Noticeably, the proposed approach -based on the multivariate Brownian distance correlation- performs extremely well compared with the optimum wrapper approach. Indeed, the obtained front is most of the time located within the repeatability range of the optimum front. 


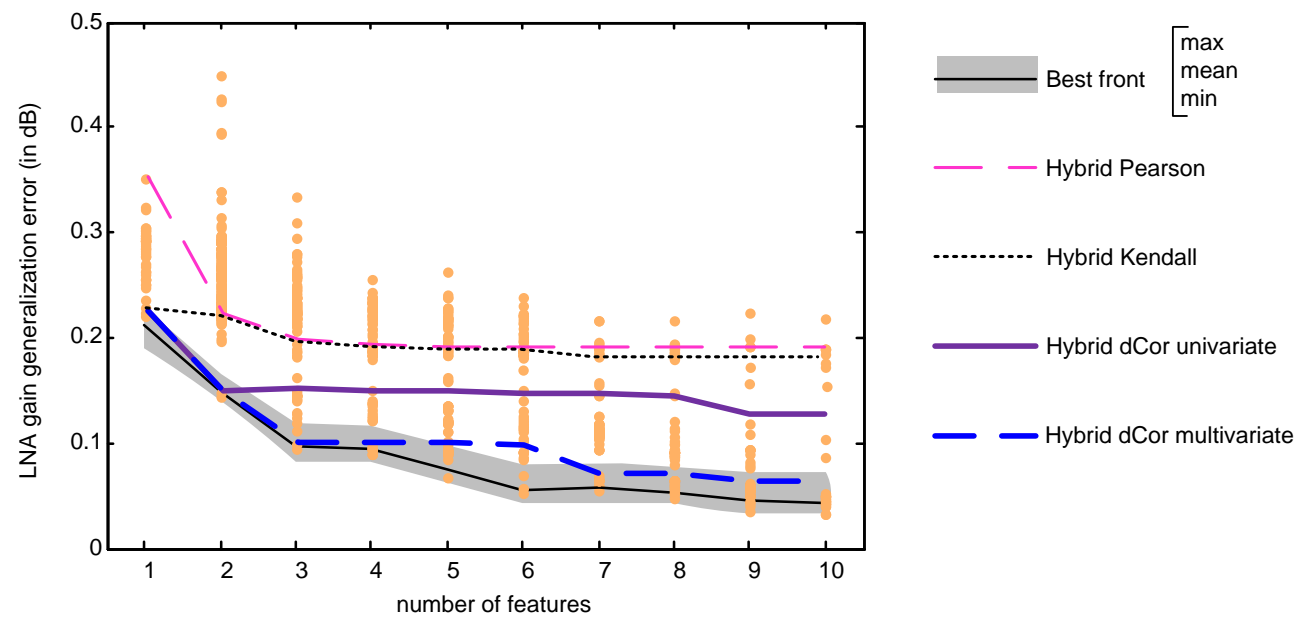

Figure 4: Generalization error for the prediction of LNA gain: Fronts obtained for correlation-directed search with different correlations and scatterplot of the explored space through wrapper approach

\subsection{Switched-Capacitor Filter}

The Device Under Test for this case study is a switched-capacitor biquad in a fullydifferential lowpass configuration. Fig. 5 shows the schematic of the DUT. A nonlinear feedback loop has been added to force the oscillation during the test mode. This feedback loop comprises a simple comparator followed by a single-bit D/A converter. The complete system has been designed in a $3.3 \mathrm{~V}-0.35 \mu \mathrm{m}$ CMOS technology for a 1 $\mathrm{MHz}$ clock frequency at nominal operation conditions.

Oscillation-based test relies in forcing oscillators either in a circuit or in a full system. The DUT is reconfigured into an oscillator, and the resulting oscillation is characterized. The key premise in this test framework is that the presence of any fault in the DUT changes its oscillation features in comparison to the fault-free circuit. Predictive OBT (POBT) has been proposed to extend traditional OBT to a functional test framework. POBT makes use of statistical post-processing tools to predict functional specifications of the DUT from its oscillation features in test mode $[25,26]$.

The oscillation frequency and the magnitudes of the first three harmonic components are used as features. Such set of features can be efficiently computed on-chip in the digital domain [27], and can be related to performance parameters of the DUT such as its frequency response and linearity figures. In addition, following the multi-condition test strategy in [6] to enhance the feature space, we repeat the test at a $75 \%$ power supply, and at a ten times higher clock frequency, leading to a total of 18 features. The goal of the machine-learning based test is to predict the Total Harmonic Distortion of the filter.

A Monte Carlo simulation varying the process parameters according to the design kit was performed to generate 200 instances. This number is relatively low for machinelearning purposes but this is due to the fact that the evaluation of the THD is relatively time-consuming. 50 instances out of these 200 were set aside for the validation set.

Figure 6 shows the results obtained for the Switched-Capacitor filter. For this case 

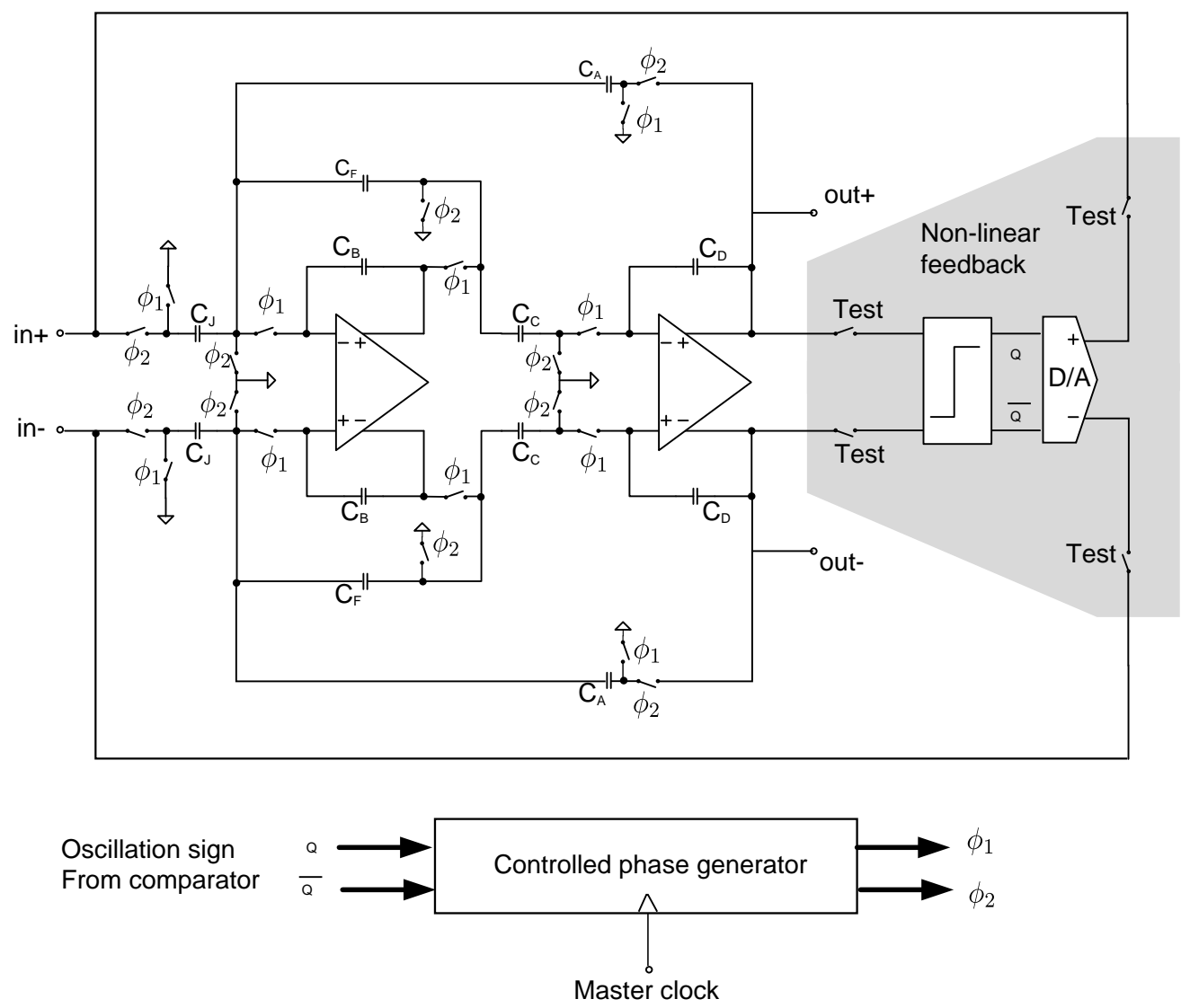

Figure 5: Schematic of the SC filter under test 


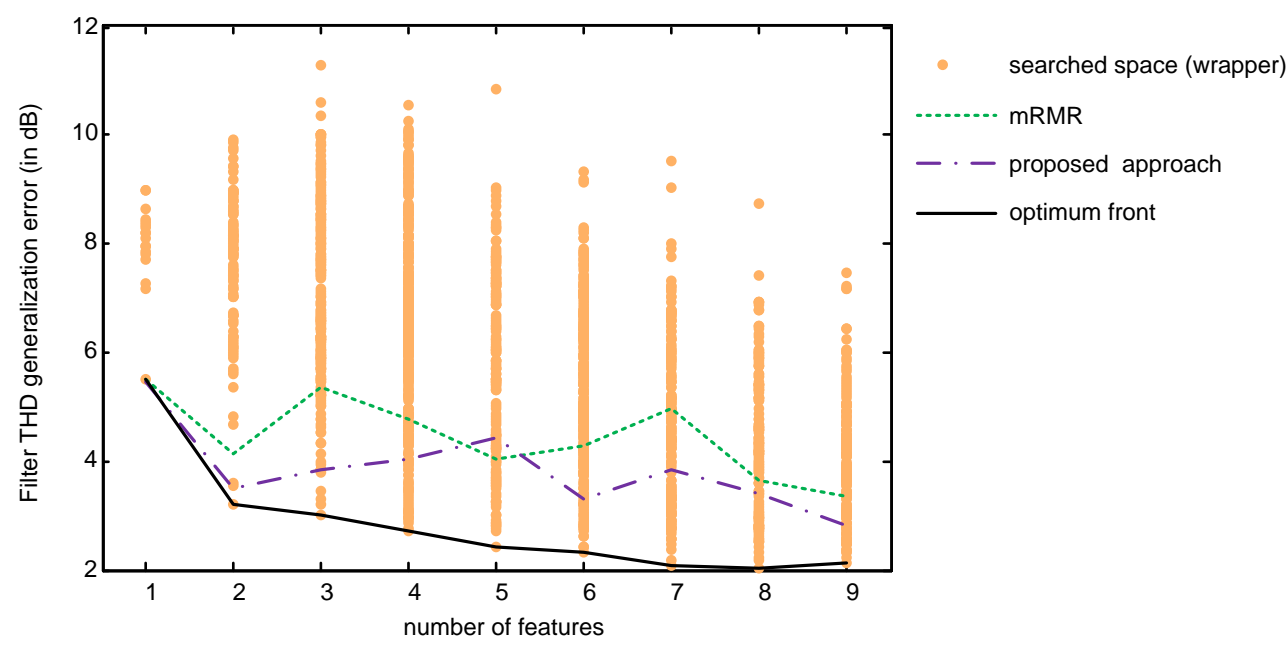

Figure 6: Generalization error for the THD of the SC filter: Fronts obtained from mRMR filtering methods, wrapper search and the proposed method

study, the proposed approach produces models that are close to the optimum front and outperforms the mRMR filtering method. For dimensions above 3, the results appear to be quite unreliable.

We believe that this is due to the fact that only few samples are available in the training set (150, actually). The repeatability of the distance correlation computation may thus not be high enough. In addition, it is well known that small sample sets are more prone to overfitting.

A simple example may ease the understanding. Suppose that you have unidimensional data $(x, y)$ generated by an unknown function $y=f(x)$ and that you want to model the relation between $x$ and $y$ through a polynomial. The degree of the polynomial can be seen as the number of features (you consider as features $x, x^{2}, x^{3}$, etc.). It is obvious that if you have $n$ samples, a polynomial of degree $n$ would lead to a perfect fit. Would you say, though, that it faithfully represents function $f$ ?

This can clearly be seen in Figure 7. It shows the THD generalization error computed on the independent validation test set together with the THD cross-validation error obtained on the training set. It can be seen how the cross-validation error steadily decreases for both the proposed approach and mRMR, while the error on the validation set remains relatively high.

Such a discrepancy between the two errors should thus be taken as an early-stopping criterion for the directed search. For this particular case of study, we can hardly discriminate between the proposed approach and the mRMR since they give similar results up to 3 features.

\subsection{Reference Voltage Generator}

The voltage reference interface depicted in Fig. 8 is used in a state-of-the-art $15 \mathrm{~b}$ pipeline converter in $0.18 \mu \mathrm{m} 1.8 \mathrm{~V}$ CMOS technology. Such an interface takes as an input the temperature-stable voltage references that are derived from a band-gap and 


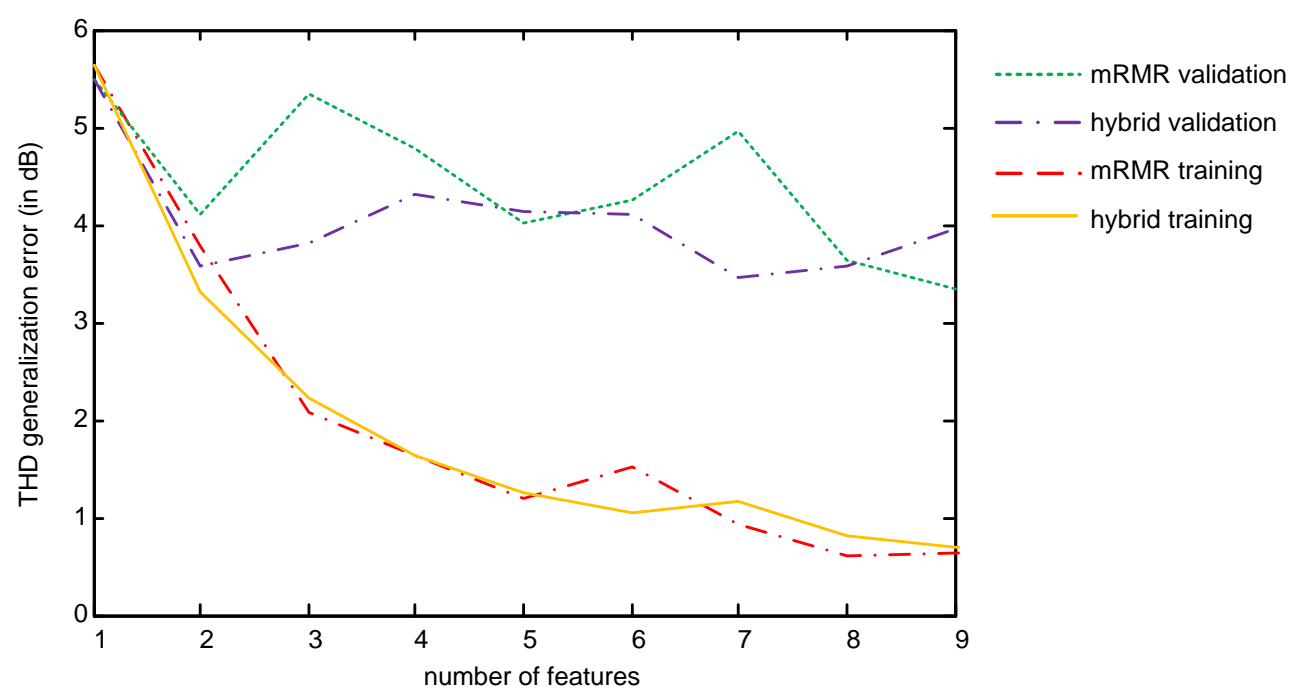

Figure 7: Overfitting for the prediction of the THD of the SC filter. Training set and validation set errors clearly diverge as model dimensionality increases

outputs the same voltage level but with a high driving capability. Actually, this circuit is designed to withstand the dynamic load of a $10 \mathrm{pF}$ sampling capacitor at $100 \mathrm{MHz}$. It consists of a series of voltage followers dimensioned to cope with the current peak. In order to avoid stability issues and slow settling, the control feedback is performed on a scaled down replica of the followers [28].

Its most important specification is that it must settle within one quarter of an LSB at $5 \mathrm{~ns}$ after the connection of a discharged capacitor. The settling error at $5 \mathrm{~ns}$ is thus the performance that the model has to predict.

For this case study, we consider as the search input space the 29 global process parameters generated by the design KIT that are varied during the Monte Carlo process simulation (the $\Delta V_{t h}$ of the different families of NMOS and PMOS transistors, their mobility variations, the resistivity of the different types of resistors, etc.). We acknowledge that it is not a realistic test case since these process parameters would not be easy to measure in practice, but it will serve the purpose of validation for the proposed technique. Since the features are the process parameters that are actually varied, we can be certain that there exist an analytical relationship between the features and the performance. An appropriate machine-learning algorithm is thus expected to provide a low generalization error. In this case 10000 Monte Carlo instances were simulated for which the settling error was measured and the 29 process parameters (i.e. the features) were stored. The data was split equally into training and validation sets of 5000 instances.

In Figure 9 the 29-dimension input space of process parameters was explored to select those that best explain the settling error of the continuous-time reference generator. It can be seen that the mRMR filtering approach in this case leads to results that are comparable with the proposed approach. The mRMR approach actually has an edge over the proposed directed search approach for low-dimensionality models but then scales worse for higher dimensionality. 


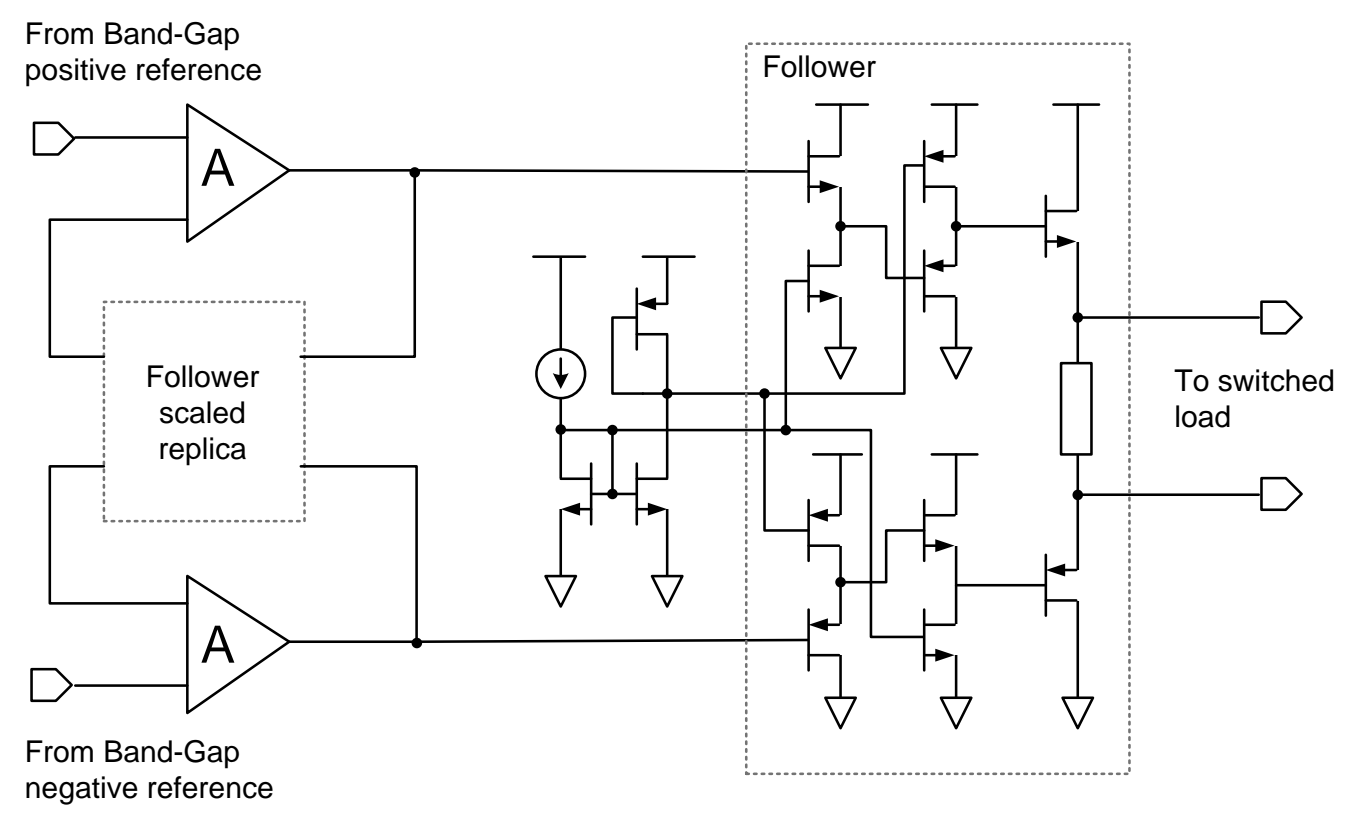

Figure 8: Schematic view of a voltage reference interface

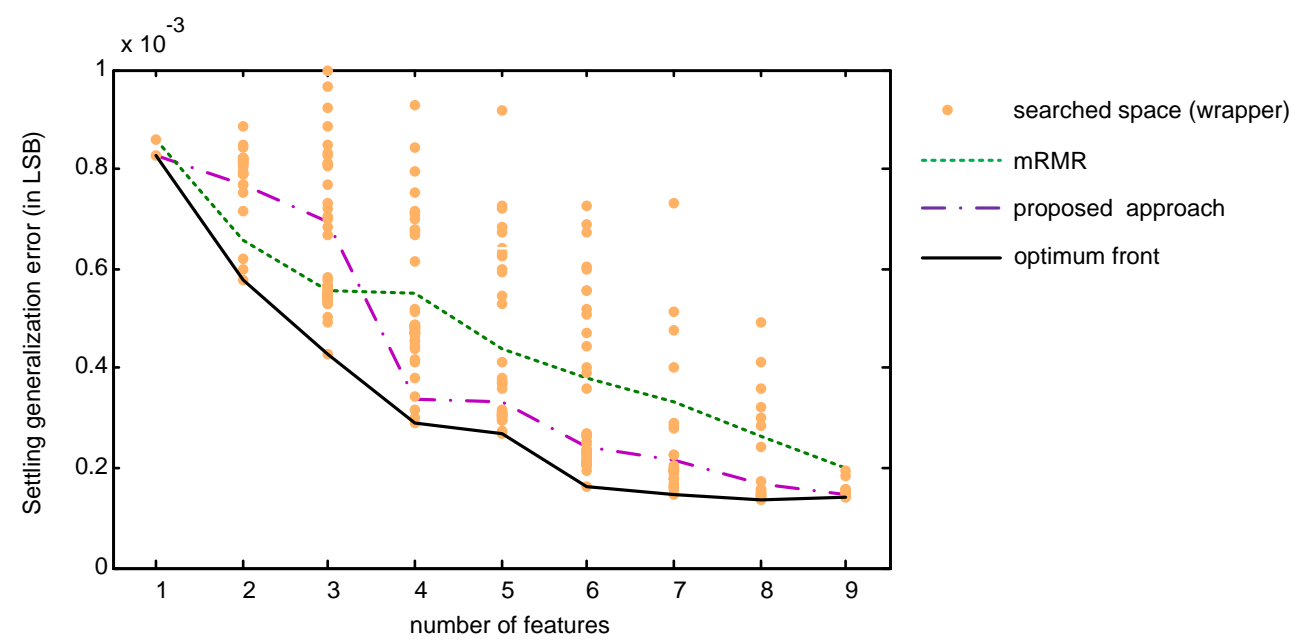

Figure 9: Generalization error for the settling error of the voltage reference generator (expressed in LSB of the ADC): Fronts obtained from mRMR filtering methods, wrapper search and the proposed method 


\section{4. $\Sigma \Delta$ modulator}

We have also re-used past work on digital tests for $\Sigma \Delta$ converters [29]. These digital tests provide simple features that can be used to feed a regression model. The digital tests that are applied to the modulator have been detailed elsewhere [29], but let us briefly recall the fundamentals. The basic idea is to re-use the feedback DAC (or add an extra 1-bit DAC) to send a digital test sequence to the modulator under test. Furthermore, the modulator can be reconfigured in smaller parts such that all the integrators can be tested. The digital sequence and the DfT configuration are chosen such that the nonideality under consideration manifests itself as a deviation in the DC component of the output bit stream. In this way, the feature can be generated with a simple counter or take advantage of the modulator decimation filter.

Several test features have been proposed but for the sake of simplicity, we only consider two of them here:

On one hand, an integrator leakage feature is obtained using a periodic sequence with mean value different from zero, and then its opposite (in order to get rid of possible offsets). More concretely the period is $\left[\begin{array}{llllll}1 & 1 & 1 & 1 & 1 & -1\end{array}\right]$ and the mean value $2 / 3$.

On the other hand, an integrator settling error feature is obtained with a sequence of zero mean value $\left[\begin{array}{llllll}1 & 1 & -1 & 1 & -1 & -1\end{array}\right]$ such that the clock period is doubled for a 1 input and remains nominal for a -1 input. Conversely, a second acquisition is performed inverting the clock dependence in order to get rid of possible offsets. For this particular test, in addition to the DC component of the output bitstream, we have to acquire the number of occurrences of the combination of a -1 input sample and a 1 feedback sample (conversely a 1 input sample and a -1 feedback sample for the second acquisition). This only requires an additional counter and a couple of logic gates.

The circuit under test is the $0.35 \mu \mathrm{m}$ CMOS switched-capacitor third order 2-1 cascaded modulator presented in [29] and depicted in Fig. 10. Simple Design-for-Testability (DfT) modifications in the control of selected switches allow performing several digital tests.

With these modifications it is actually possible to obtain the leakage and settling test features independently on each of the three amplifiers. Initially, we thus have a 6 dimension input space that we want to map onto the unidimensional output space formed by the SNDR. In addition, again using a multi-condition strategy [6], we repeat the six tests at $80 \%$ of the power supply in order to stimulate the limited output range of the amplifiers and the settling tests at $120 \%$ of the nominal frequency in order to observe more important settling errors (we do not repeat the leakage tests in this case because we do not expect any change). In this way, the dimension of the input space grows to 15.

Ideally, we should thus perform an electrical Monte-Carlo simulation of a $\Sigma \Delta$ modulator for the proposed digital tests as well as for the functional test. Unfortunately, such simulations are not feasible on our hardware because even a single simulation to measure the SNDR of an instance already takes days to complete. To circumvent this issue we instead perform a Monte-Carlo simulation of 300 instances of the amplifiers present in the modulator and extract their main parameters (namely, gain, output range, linearity, bandwidth, and slew-rate), at two power supply voltages (the nominal voltage $3.3 \mathrm{~V}$ and a reduced voltage of $2.7 \mathrm{~V}$ ). The extracted parameters are then used in a high-level model similar to those proposed in [30]. In this way, we do not have the exact details of the 


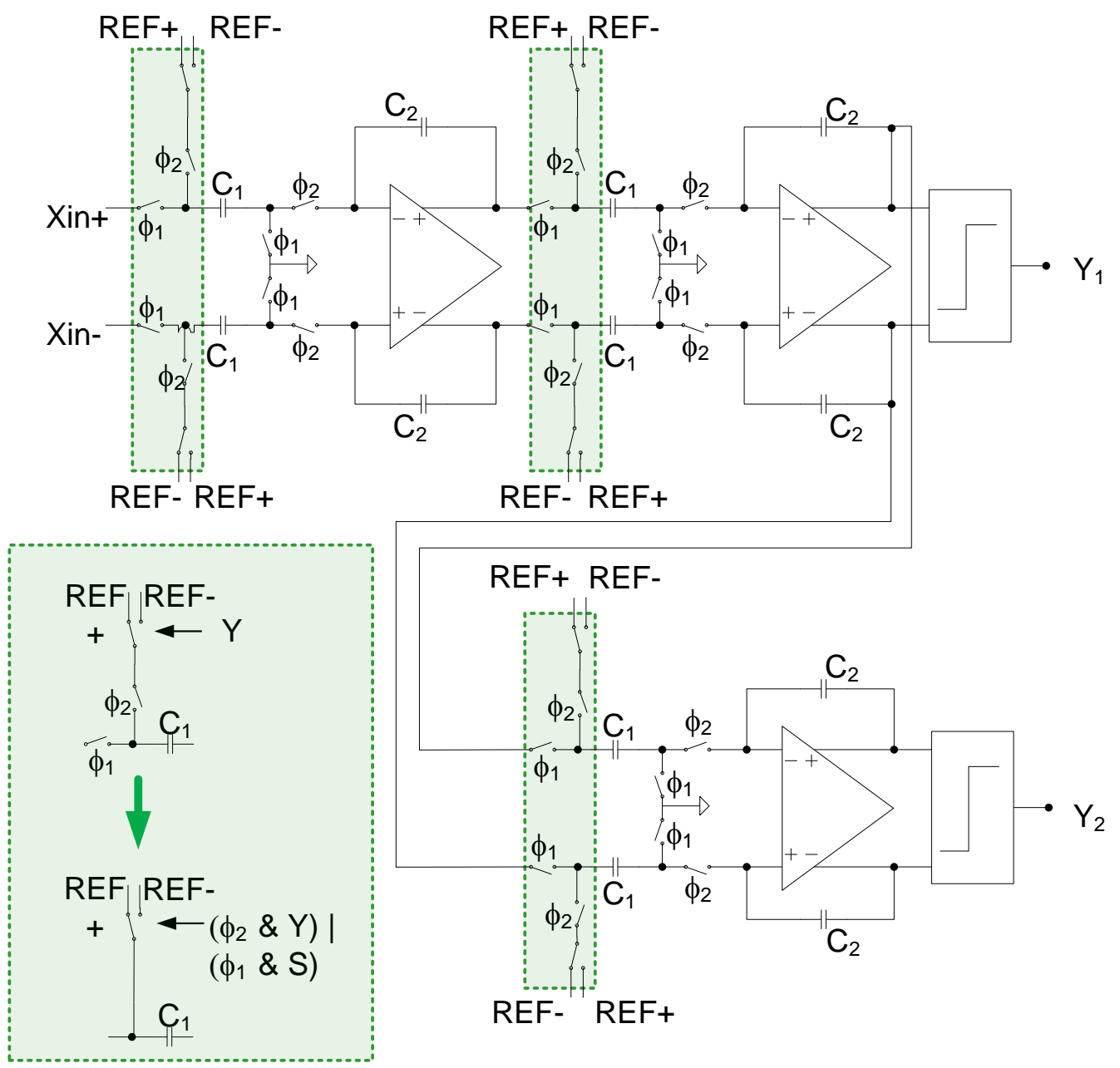

Figure 10: Schematic of the modulator under test. Modified test-mode switch control: i) Nominal input is disconnected ii) Feedback DAC is connected during sampling phase iii) The desired sequence $\mathrm{S}$ is sent to the integrator 


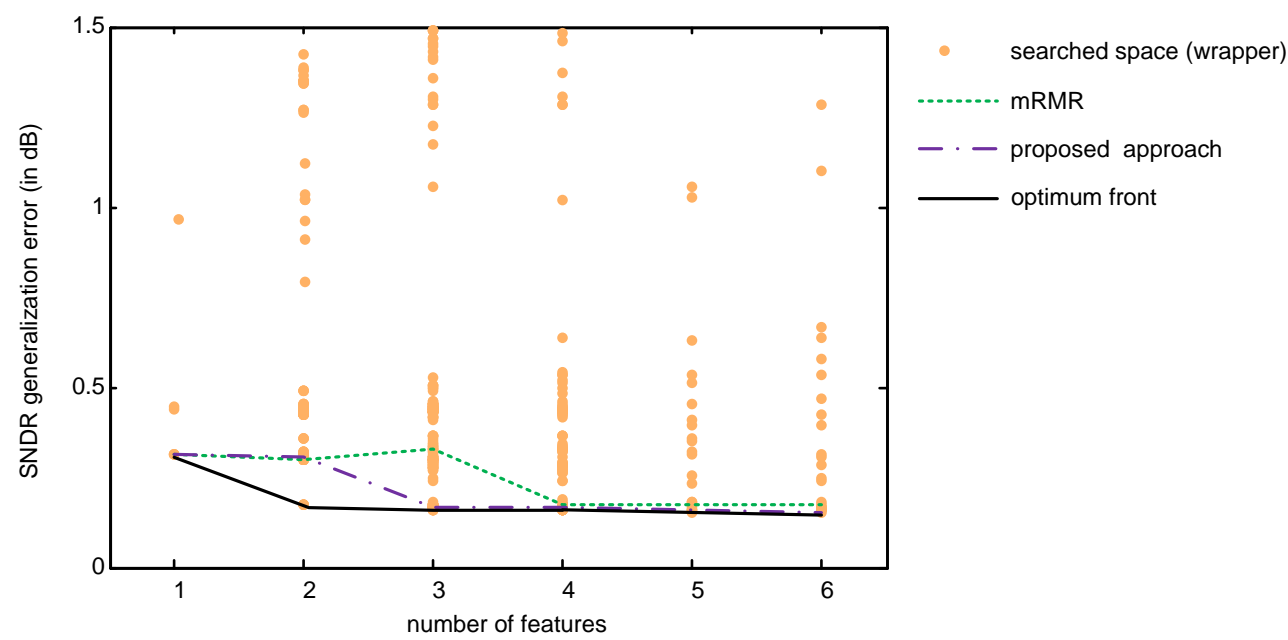

Figure 11: Generalization error for the SNDR of the $\Sigma \Delta$ filter: Fronts obtained from mRMR filtering methods, wrapper search and the proposed method

electrical simulation, but the correlation between the results at different power supplies is not artificial. It corresponds to real process variations. Notice that the frequency stress is applied at behavioral level directly and does not require a different Monte-Carlo simulation of the amplifiers.

Figure 11 displays the performance of the proposed feature selection method for the system-level case of the third order $\Sigma \Delta$ modulator. Here both the mRMR filtering method and the proposed directed search approach give very similar results that are very close to the optimum front. By the way, both only fail to identify the second best feature (This again may be due to the relatively limited number of samples in the training set) but notice that the selected point is actually the second best according to the wrapper search. We should also remark that the optimum front is almost flat: an optimum model is obtained with only two features. After diagnosing the modulator, it comes that the design was significantly guard-banded against integrator leakage while its dynamic behavior (i.e. the settling of the integrators) was pushed much closer to the viability limit. As a result, a good prediction could already be obtained by the digital tests that predict settling errors. In addition, the same amplifiers are used in the three integrators. As process variations are the dominant variability causes, the three settling error features corresponding to the three integrators bring an almost perfectly redundant information. Under this scenario, a fairly good model can be derived with only one of these features and additional ones only bring small refinement. In any case, we can say that the proposed method performs as expected.

\subsection{Machine-learning repository dataset}

Finally, we have also apply the proposed approach to the "YearPredictionMSD" dataset that is publicly available at [31]. This dataset has actually nothing to do with Alternate Test, not even with electronics. The objective is to predict the year of production of a song as a function of 90 different parameters related to the timbre of the songs. 


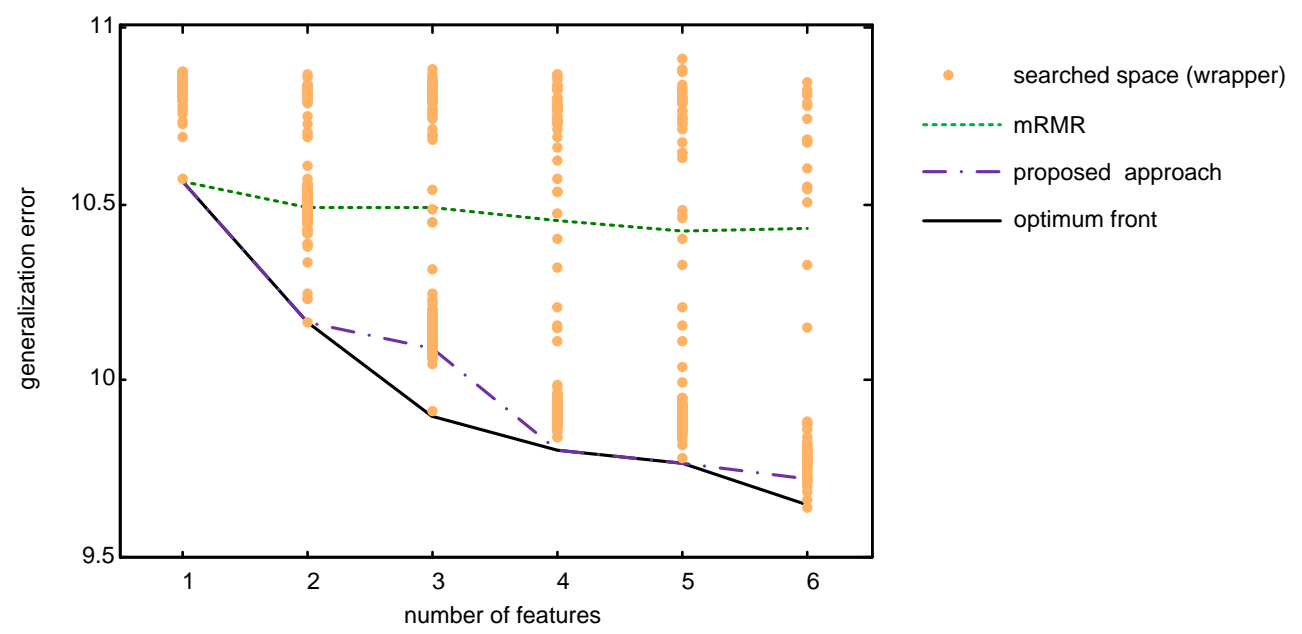

Figure 12: Generalization error for song year prediction: Fronts obtained from mRMR filtering method and the proposed directed search together with the scatterplot of combinations explored by wrapper search

Table 3: Summary of feature selection performance on the cases of study

\begin{tabular}{lcccc}
\hline case & \# feat. & \# samples & $F O M_{m R M R}$ & $F O M_{\text {proposed }}$ \\
\hline RF LNA & 42 & 2000 & 9.7 & 1.3 \\
SC filter & 18 & 200 & 2.5 & 1.6 \\
Reference Buffer & 29 & 10000 & 12.5 & 7.5 \\
$\Sigma \Delta$ modulator & 15 & 300 & 3 & 1.4 \\
dataset & 90 & 66000 & 37.4 & 2.5 \\
\hline
\end{tabular}

This dataset is included in this paper to illustrate that the proposed feature selection method is actually not restricted to a Alternate Test and could be used in other domains.

The amount of data available for training is huge (more than 500000) so we deliberately restricted the training set to 10000 instances (i.e. songs). However, the test set is the one proposed in the dataset description since model evaluation is orders of magnitude faster than model training.

Figure 12 shows that the proposed method clearly outperforms the mRMR approach, producing a front that is very close to the best front obtained though stepwise wrapper search. It is worth noticing that the model prediction is not very accurate, with a generalization error around 10 years for songs ranging from 1922 to 2011. Despite of this and the high dimensionality of the search space, the proposed method is able to draw the most out of the available information.

\subsection{Results summary}

Table 3 summarizes the obtained results for the different cases of study. For the sake of brevity, we concentrate the information about the generated fronts into a single Figure 
of Merit:

$$
F O M=\frac{1 / n \sum_{i=1}^{n} e r r_{\text {method }}(i)-e r r_{\text {opt }}(i)}{\sigma_{\text {rep }}}
$$

The average distance of the selected method generalization error ( $\left.e r r_{m e t h o d}\right)$ to that of the optimum front $\left(e r r_{o p t}\right)$ over the different number of features $(n)$, expressed as a fraction of the repeatability of the optimum front $\left(\sigma_{r e p}\right)$. This repeatability is estimated by repeating the model training and subsequent generalization error computation 30 times on different random splits of the data, as explained in section 3.1. Obviously, the lower the FOM the better the feature selection.

\section{Conclusions}

This work proposes a computationally efficient method for feature selection based on a correlation-directed search approach. Relying on multivariate Brownian Distance Correlation, which compute the correlation between pairwise distances in the input space (the feature space) and the output space (the performance space), each iteration of the search algorithm selects the feature that best correlates to the residues of the previous fit.

The proposed algorithm has been validated for several cases of study: an RF LNA, a Switched-Capacitor filter with Oscillation-Based Test, a voltage reference buffer for a high-speed ADC, a third-order cascaded 2-1 $\Sigma \Delta$ modulator and a third-party dataset.

We can conclude that the proposed Brownian Distance Correlation-directed search outperforms the best reviewed filtering method (this can be concluded essentially form the first case of study) and always provides models that are close to the optimum fronts obtained by wrapper search at a fraction of the computational cost (the gain factor over stepwise wrapper search is approximately equal to the number of features in the search space). It is thus a reliable candidate for efficient feature selection that can be used to quickly identify appropriate features sets to perform Alternate Test at a reduced computational cost.

\section{Acknowledgment}

This work has been partially funded by the Spanish Government project TEC201568448-R (co-funded by FEDER program) and supported by the LabEx PERSYVAL-Lab (ANR-11-LABX-0025-01) funded by the French program Investissement d'avenir

[1] P. N. Variyam and A. Chatterjee, "Enhancing test effectiveness for analog circuits using synthesized measurements," in Proc. of the IEEE VLSI Test Symposium, 1998, pp. 132-137.

[2] K. Huang, N. Kupp, J. M. Carulli, and Y. Makris, "Handling discontinuous effects in modeling spatial correlation of wafer-level analog/RF tests," in Pr0c. of Design, Automation Test in Europe Conference (DATE), 2013, pp. 553-558.

[3] R. Voorakaranam, S. S. Akbay, S. Bhattacharya, S. Cherubal, and A. Chatterjee, "Signature testing of analog and RF circuits: Algorithms and methodology," IEEE Transactions on Circuits and Systems I: Regular Papers, vol. 54, no. 5, pp. 1018-1031, 2007.

[4] A. Halder, S. Bhattacharya, and A. Chatterjee, "Automatic multitone alternate test generation for RF circuits using behavioral models," in Proc. of International Test Conference, 2003.

[5] N. Kupp, P. Drineas, M. Slamani, and Y. Makris, "On boosting the accuracy of non-RF to RF correlation-based specification test compaction," Journal of Electronic Testing, vol. 25, no. 6, pp. 309-321, 2009. 
[6] M. J. Barragan, G. Leger, and J. L. Huertas, "Multi-condition alternate test of analog, mixed-signal, and rf systems," Proc. of Latin American Test Workshop (LATW), pp. 1-6, 2012.

[7] G. Leger and M. Barragan, "A hybrid method for feature selection in the context of alternate test," in International Conference on Synthesis, Modeling, Analysis and Simulation Methods and Applications to Circuit Design (SMACD), Sep. 2015, pp. 1-4.

[8] I. Guyon and A. Elisseeff, "An introduction to variable and feature selection," Journal of Machine Learning Research, vol. 3, Mar. 2003.

[9] L. Milor and A. L. Sangiovanni-Vincentelli, "Minimizing production test time to detect faults in analog circuits," IEEE Transactions on Computer-Aided Design of Integrated Circuits and Systems, vol. 13, no. 6, pp. 796-813, 1994.

[10] J. B. Brockman and S. W. Director, "Predictive subset testing: optimizing IC parametric performance testing for quality, cost, and yield," IEEE Transactions on Semiconductor Manufacturing, vol. 2, no. 3, pp. 104-113, Aug. 1989.

[11] H.-G. Stratigopoulos and Y. Makris, "Nonlinear decision boundaries for testing analog circuits," Transactions on Computer-Aided Design of Integrated Circuits and Systems, vol. 24, no. 11, pp. 1760-1773, 2005.

[12] J. D. Wichard, M. J. Ogorzalek, and C. Merkwirth, "Entool-a toolbox for ensemble modelling," in Europhysics Conference Abstracts ECA, vol. 27, 2003.

[13] S. Biswas, P. Li, R. D. Blanton, and L. T. Pileggi, "Specification test compaction for analog circuits and mems [accelerometer and opamp examples]," in Design, Automation and Test in Europe, 2005. Proceedings, 2005, pp. 164-169.

[14] R. Kohavi and G. H. John, "Wrappers for feature subset selection," Artificial intelligence, vol. 97, no. $1,1997$.

[15] P. Mordohai and G. Medioni, "Dimensionality estimation, manifold learning and function approximation using tensor voting," The Journal of Machine Learning Research, vol. 11, pp. 411-450, 2010

[16] V. Bolón-Canedo, N. Sánchez-Maroño, and A. Alonso-Betanzos, "A review of feature selection methods on synthetic data," Knowledge and Information Systems, vol. 34, no. 3, pp. 483-519, Mar. 2013

[17] H. Peng, F. Long, and C. Ding, "Feature selection based on mutual information criteria of maxdependency, max-relevance, and min-redundancy," IEEE Trans. on Pattern Analysis and Machine Intelligence, vol. 27, no. 8, pp. 1226-1238, 2005

[18] H. Ayari et al., "Smart selection of indirect parameters for DC-based alternate RF IC testing," VLSI Test Symposium, pp. 19-24, 2012.

[19] M. Barragan and G. Leger, "Efficient selection of signatures for analog/RF alternate test," in Proc. of IEEE European Test Symposium (ETS), pp. 1-6, May 2013.

[20] G. J. Székely and M. L. Rizzo, "Brownian distance covariance," The Annals of Applied Statistics, vol. 3, no. 4, pp. 1236-1265, Dec. 2009.

[21] A. Gomez-Pau, L. Balado, and J. Figueras, "Quality metrics for mixed-signal indirect testing," in IEEE Conference on Design of Circuits and Integrated Circuits (DCIS), pp. 1-6, 2014.

[22] — , "Efficient Production Binning Using Octree Tessellation in the Alternate Measurements Space," IEEE Transactions on Computer-Aided Design of Integrated Circuits and Systems, vol. PP, no. 99 , pp. 1-1, 2015.

[23] H. G. Stratigopoulos et al., "RF Specification Test Compaction Using Learning Machines," Transactions on Very Large Scale Integration (VLSI) Systems, vol. 18, no. 6, pp. 998-1002, June 2010.

[24] M. J. Barragán et al., "Alternate test of lnas through ensemble learning of on-chip digital envelope signatures," Journal of Electronic Testing, vol. 27, no. 3, pp. 277-288, 2011.

[25] G. Huertas, D. Vazquez, E. Peralias, A. Rueda, and J. Huertas, "Practical oscillation-based test of integrated filters," IEEE Design Test of Computers, vol. 19, no. 6, pp. $64-72$, nov/dec 2002.

[26] A. Raghunathan, H. J. Shin, J. Abraham, and A. Chatterjee, "Prediction of analog performance parameters using oscillation based test," in Proc. of the IEEE VLSI Test Symposium, april 2004, pp. $377-382$.

[27] D. Vazquez, G. Huertas, A. Luque, M. J. Barragan, G. Leger, A. Rueda, and J. L. Huertas, "SineWave signal characterization using Square-Wave and $\Sigma \Delta$-Modulation: application to Mixed-Signal BIST," Journal of Electronic Testing, vol. 21, no. 3, pp. 221-232, 2005.

[28] C.-k. Lee, W. Kim, H. Kang, and S.-T. Ryu, "A Replica-Driving Technique for High Performance SC Circuits and Pipelined ADC Design," IEEE Trans. on Circuits and Systems II: Express Briefs, vol. 60 , no. 9 , pp. 557-561, 2013.

[29] G. Leger and A. Rueda, "Low-Cost digital detection of parametric faults in cascaded $\Sigma \Delta$ modula- 
tors," IEEE Transactions on Circuits and Systems I: Regular Papers, vol. 56, no. 7, pp. 1326-1338, 2009.

[30] P. Malcovati, S. Brigati, F. Francesconi, F. Maloberti, P. Cusinato, and A. Baschirotto, "Behavioral modeling of switched-capacitor sigma-delta modulators," IEEE Trans. on Circuits and Systems I: Fundamental Theory and Applications, vol. 50, no. 3, pp. 352-364, 2003.

[31] M. Lichman, "UCI machine learning repository," 2013. [Online]. Available: http://archive.ics.uci.edu/ml 